\title{
Image Segmentation using Canny Edge and finding the Tumor Area in Image using Hierarchical Clustering
}

\author{
Bandana Bali \\ Ph.D. Research Scholar \\ Computer Science \& Engineering Department \\ Uttrakhand Technical University, Dehradun
}

\author{
Brij Mohan Singh \\ Associate Professor and Head, \\ Department of Information and Technology \\ College of Engineering, Roorkee
}

\begin{abstract}
Image segmentation of Brain MRI holds great significance in the determination of valuable functional and anatomical information of a disease like tumors. It not only advances the diagnostic techniques but also proves to be of enormous facilitation in the planning of treatment. In this research paper, we will be utilizing the bilateral filter technique to eliminate noise from the brain magnetic resonance imaging images, following by applying the improved canny edge detection algorithm for image segmentation to locate the ridges of tumor areas in them. The last step of hierarchical clustering algorithm application will aid in highlighting the affected area in the images thereby addressing the issues of clear location of tumor cells in the brain MRI images.
\end{abstract}

\section{Keywords}

Brain, MRI, Magnetic Resonance Imaging, Segmentation, Algorithm, Tumor, Highlight.

\section{INTRODUCTION}

Segmentation of an image is a highly demanding procedure that basically converts a digital image into numerous segments for simplification. The idea behind image segmentation is to make it simpler for individuals to clearly and easily understand and analyze it for better planning. In other words, the segmentation of a digital image includes a set of segments that are jointly utilized to evidently and unmistakably determine the objects or lines or curves in it.

Last decade witnessed a lot of progressive development in the field of brain imaging technologies in order to assist the medical world with simpler and quick analysis of brain anatomy and its functions. The rising issues of brain tumors and brain injuries have further boosted this development. With majority of the medical practitioners utilizing the magnetic resonance imaging technique (MRI) to access and detect the brain and its anatomy, brain MRI has become a fad in the medical world. However, for a common man, understanding and depicting the MRI images can prove to be tedious task. Therefore, image segmentation of the brain MRI proves to be of great help not for the medical practitioners in saving time to analyse the brain for functional and anatomical information but also for the laymen to understand it without much hassle.

MRI comprises of large datasets of high quality that consumes a lot of time in determining and analyzing them. Segmentation of the brain MRI images simplify the whole process, thereby giving more time to the medical practitioners to utilize it in the better treatment planning.

MRI segmentation is a significant process which is used to accurately segment the anatomical regions of the brain in order to extract vital information from it. The process is generally used in evaluating and envisioning the different structures of the brain, outlining the abrasions or tumors, analyzing its development, and image-guided interferences to properly plan surgeries or treatments.

Brain tumor is considered as one of the deadliest diseases in the world is hard to detect in the early stages, which reduces the chances of survival of the particular human being. Although the large and complex MRI segmentation makes it possible to detect it in its early stages but the time consumed in analyzing the brain structures and the affected areas was a big drawback. The latest development in this field has enhanced the analysis for functional and anatomical data. Brain MRI technique is commonly used these days to envisage the structure of the brain and its affected area to decide on the treatment procedure. However, the detection of a tumor entails a list of steps to clearly process the MRI image which includes the preprocessing of the image, extraction of its features, image enhancements and then its classification to conclude whether the person has tumor or not.

MRI being an advanced technique facilitates the medical practitioners with advanced data on brain structures, its soft tissues and its affected areas, thereby giving enough time to the patient to start the right treatment at the right time. Despite its being an advanced technology, brain MRI technology has its set of limitations. To overcome these issues, we are proposing a novel algorithm that will not only enhance the image of the brain MRI but also make it easier for all the individuals irrespective of their being a medical practitioner or not to comprehend and locate the tumor affected area in the brain.

\section{LITERATURE SURVEY}

Brain Tumor detection and MRI segmentation techniques have allured lots of attention from the researchers around the world. Many algorithms have been proposed from time to time to improve and enhance the tumor detection techniques. Despite that, the field still asked for better algorithms to get satisfactory results to plan better treatment therapies. Research studies of a few of researchers in this field are mention below.

Vasupradha Vijay et al. [1] in their research study "Automated Brain Tumor Segmentation and Detection in MRI using Enhanced Darwinian Particle Swarm Optimization (EDPSO)" proposed Enhanced Darwinian Particle Swarm Optimization (EDPSO) for automated tumor segmentation which overcome the drawback of existing Particle Swarm Optimization(PSO). This innovative method consisted of four steps, wherein pre-processing, film artifacts and unwanted portions of MRI images were removed using tracking 
algorithm, followed by removal of noises and high frequency component with the help of Gaussian filter. The third step covered segmentation by using Darwinian Particle Swarm Optimization and the last step involved classification by Adaptive Neuro Fuzzy Inference System. The performance of the proposed method was systematically evaluated using the MRI brain images.

Hala Ali et al. [2] in their research paper "Multi-resolution MRI Brain Image Segmentation Based on Morphological Pyramid and Fuzzy C-mean Clustering" proposed a new system for a multi-resolution MRI brain image segmentation, which was based on a morphological pyramid with fuzzy Cmean (FCM) clustering. They compared their proposed system with some state of the art segmentation techniques on two different brain data sets. Experimental results showed that the proposed system improved the accuracy of the MRI brain image segmentation.

Archana $\mathrm{M}$ et al. [3] in their research study "A Hybrid Approach to Automated Delineation of Brain Tissue in Alzheimer MR Images" described a methodology to combine a modified level set framework with Otsu algorithm for automatic and accurate segmentation of brain tissue and better approximation of initial contour. MR images used were acquired from open source online database OASIS. A mask image was obtained by performing Otsu thresholding on the MR image and the maximum connected region in the mask was given as the initial contour to the level set framework. A distance regularization term was included in the energy optimization function of the level set in order to maintain stability of the evolving contour. The results of segmentation were validated by comparing with the ground truth using similarity measures.

Jothi G. et al. [4] in their research work "Hybrid Tolerance Rough Set-Firefly based supervised feature selection for MRI brain tumor image classification" a hybridization of two techniques, Tolerance Rough Set (TRS) and Firefly Algorithm (FA) were used to select the imperative features of brain tumor. Performance of TRSFFQR was compared with Artificial Bee Colony (ABC), Cuckoo Search Algorithm (CSA), Supervised Tolerance Rough Set-PSO based Relative Reduct (STRSPSO-RR) and Supervised Tolerance Rough Set-PSO based Quick Reduct (STRSPSO-QR). The experimental result showed the effectiveness of the proposed technique as well as improvements over the existing supervised feature selection algorithms.

S. Jansi et al. [5] in their research paper "Modified FCM using Genetic Algorithm for Segmentation of MRI Brain Images" concentrated on clustering methods, especially $\mathrm{K}$ Means, Fuzzy C-Means clustering algorithm for segmentation of Gray Matter, White Matter and Cerebrospinal Fluid tissues in MRI brain images. FCM was more effective to the fuzzy boundary region segment, but the biggest disadvantage was that there was no better way to find the centroid clustering value. So it would converge to the local minimum point easily. To overcome this limitation, a Genetic Algorithm was integrated along with Fuzzy Clustering Method for determining the global centroid value. Experimental outcome showed Genetic Algorithm based FCM segmentation gave better performance compared with existing methods by using evaluation metrics such as Under Segmentation, Over Segmentation and Incorrect Segmentation.

Yamini Sharma et al. [6] in their research study "Brain Tumor Extraction From MRI Image Using Mathematical Morphological Reconstruction" described an approach based on mathematical morphological reconstruction for extraction of a tumor from labeled brain magnetic resonance imaging (MRI) images affected by impulse noise. As a part of preprocessing, global thresholding technique was applied on the input MRI image. Afterward, mathematical morphological reconstruction operation segments a brain tumor from the processed image. Algorithm was further modified for nonuniform intensity regions of the brain tumor. Optionally, mathematical morphological operator was utilized to remove pepper noise if presented in tumor region. Finally, tumor region was extracted using resultant image and input MRI image. Experimental results showed the proposed method effectively extracted a brain tumor from noisy labeled MRI images.

Luciano Nieddu et al. [7] in their research work "Automatic $3 D$ Image Segmentation Using Adaptive k-means on Brain MRI" presented a fully automatic adaptive k-means segmentation algorithm for MR Images in a 3D space. They modelled the gray scale values of the $3 \mathrm{D}$ image with a White Gaussian Process and superimposed a prior model on the region process in the form of Markov Random Field. The Hammersley-Clifford theorem allowed them to model the region process in term of a Gibbs Distribution. The Gibbs parameter $\beta$ was estimated using a correlation-based technique. The segmentation was obtained maximizing the a posterior density function using an Iterated Conditional Modes technique.

Soham Sarkar et al. [8] in their research study "Multi-Level Thresholding with a Decomposition-based Multi- Objective Evolutionary Algorithm for Segmenting Natural and Medical Images" presented an alternative approach for unsupervised segmentation of natural and medical images to improve the separation between objects in the framework of multiobjective optimization. The outcome of the proposed method was evaluated by comparing against the results of other well cited algorithms both qualitatively and quantitatively on testsuites comprising well-known natural and medical test images in order to showcase the efficiency of the proposed algorithm.

JiHoon Kim et al. [9] in their research work "Using a Method Based on a Modified K-Means Clustering and Mean Shift Segmentation to Reduce File Sizes and Detect Brain Tumors from Magnetic" proposed a method of elaborating and detecting brain tumor from MRI suitable for information sharing via the internet for a healthcare provider. This method allowed for reducing image sizes without reducing the information content of the images in terms of detecting tumors. The proposed method was compared with two wellknown methods namely the conventional K-mean clustering and Fuzzy C Means (FCM) clustering. Their method detected more brain tumors than the conventional K-means clustering and FCM clustering methods and was able to provide for an efficient image data processing with reduced file sizes.

\section{PROBLEM STATEMENTS}

In our research paper, we will be removing the noise from Brain MRI Images first with the help of bilateral filter technique. This step would be followed by the application of canny edge detection algorithm to segment the images in order to find the ridges of tumor area inside the images. Finally, a hierarchical clustering algorithm will be used to highlight the area of brain tumor in the images. 


\section{RESULTS}

File compression

File size Existing Proposed

\begin{tabular}{|l|l|l|}
\hline $18.8 \mathrm{~Kb}$ & $20 \%$ & $60 \%$ \\
\hline $36 \mathrm{~Kb}$ & $85 \%$ & $89 \%$ \\
\hline $84.55 \mathrm{~Kb}$ & $10 \%$ & $68 \%$ \\
\hline
\end{tabular}

\section{Precision}

File size Existing Proposed

\begin{tabular}{|l|l|l|}
\hline $18.8 \mathrm{~Kb}$ & $0.94 \%$ & $0.95 \%$ \\
\hline $36 \mathrm{~Kb}$ & $0.98 \%$ & $0.99 \%$ \\
\hline $84.55 \mathrm{~Kb}$ & $0.97 \%$ & $0.98 \%$ \\
\hline
\end{tabular}

Recall

File Size Existing Proposed

\begin{tabular}{|l|l|l|}
\hline $18.8 \%$ & $0.99 \%$ & $0.999 \%$ \\
\hline $36.6 \%$ & $0.999 \%$ & $1 \%$ \\
\hline $84.55 \%$ & $0.98 \%$ & $0.97 \%$ \\
\hline
\end{tabular}

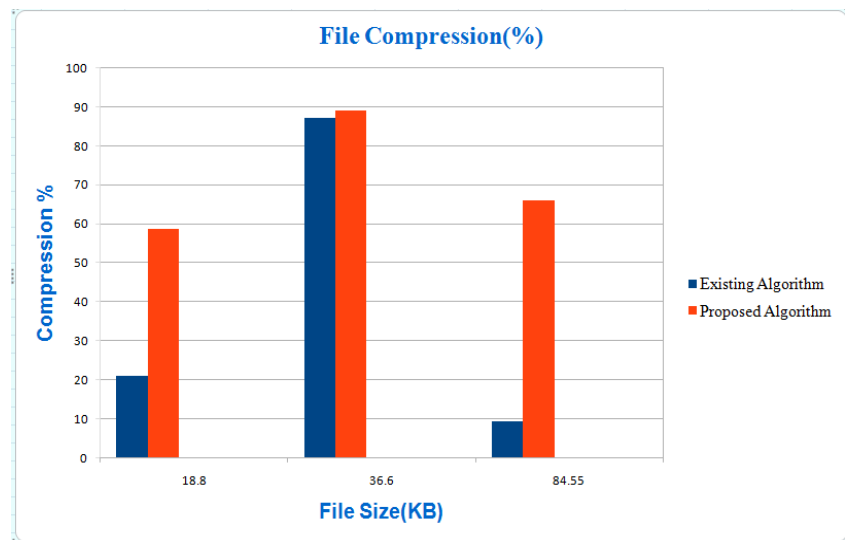

Figure 1

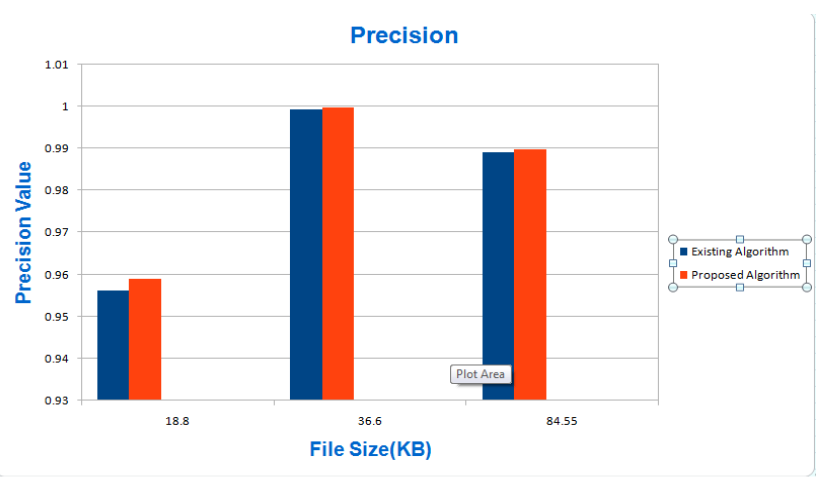

Figure 2

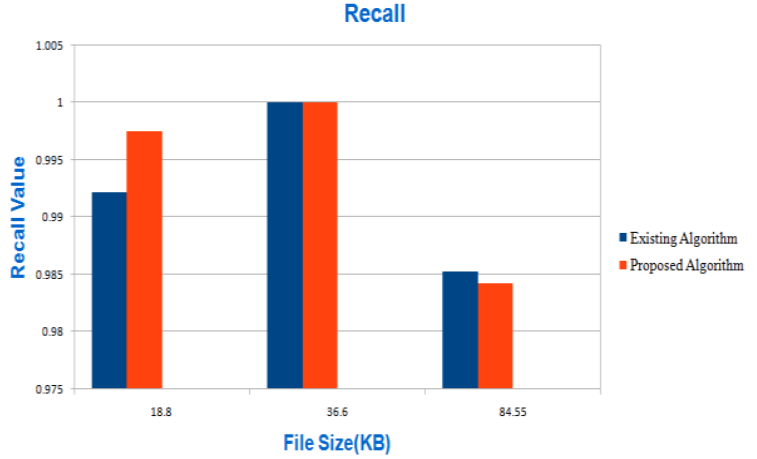

Figure 3

\section{CONCLUSION}

In this research paper, we worked upon the image segmentation of the brain MRI wherein we made use of the bilateral filter technique to remove the noise from the brain MRI image. In the second step, we applied the canny edge detection algorithm for image segmentation to determine the ridges of tumor area in the image. In the third and final step, we put into use the hierarchical clustering algorithm to highlight the area of brain tumor in the images.

\section{REFERENCES}

[1] Vasupradha Vijay et al. "Automated Brain Tumor Segmentation and Detection in MRI using Enhanced Darwinian Particle Swarm Optimization (EDPSO)" (C) 2016 Published by Elsevier B.V.

[2] Hala Ali et al. "Multi-resolution MRI Brain Image Segmentation Based on Morphological Pyramid and Fuzzy C-mean Clustering”. Arab J Sci Eng. DOI 10.1007/s13369-015-1791-x.

[3] Archana $\mathrm{M}$ et al. "A Hybrid Approach to Automated Delineation of Brain Tissue in Alzheimer MR Images". 978-0-7695-4964-4/13 \$26.00 (C) 2013 IEEE. DOI 10.1109/NEBEC.2013.39.

[4] Jothi G. et al. "Hybrid Tolerance Rough Set-Firefly based supervised feature selection for MRI brain tumor image classification". http://dx.doi.org/10.1016/ j.asoc.2016.03.014. 1568-4946/@ 2016 Elsevier B.V. All rights reserved.

[5] S. Jansi et al. "Modified FCM using Genetic Algorithm for Segmentation of MRI Brain Images". 978-1-47993975-6/14/\$31.00 @2014 IEEE. 2014 IEEE International Conference on Computational Intelligence and Computing Research.

[6] Yamini Sharma et al. "Brain Tumor Extraction From MRI Image Using Mathematical Morphological Reconstruction”. 978-1-4799-6986-9/14/\$31.00@2014 IEEE.

[7] Luciano Nieddu et al. "Automatic 3D Image Segmentation Using Adaptive k-means on Brain MRI". A. Abd Manaf et al. (Eds.): ICIEIS 2011, Part II, CCIS 252, pp. 171-183, 2011. c Springer-Verlag Berlin Heidelberg 2011.

[8] Soham Sarkar et al. "Multi-Level Thresholding with a Decomposition-based Multi- Objective Evolutionary Algorithm for Segmenting Natural and Medical Images". DOI: http://dx.doi.org/doi:10.1016/j.asoc.2016.10.032. 
[9] Ji Hoon Kim et al. in their research work "Using a Method Based on a Modified K-Means Clustering and Mean Shift Segmentation to Reduce File Sizes and Detect Brain Tumors from Magnetic". DOI 10.1007/s11277-016-3420-8. Springer Science+Business Media New York 2016.

[10] D. De Sieno, "Adding a conscience to competitive learning", Proceeding of IEEE the Second International Conference on Neural networks(ICNN88), vol. 1, pp. 117-124, 1988

[11] D. E. Goldberg, Genetic Algorithms in Search Optimization and Machine Learning, Addison Wesley Longman Pte. Ltd., pp. 60-68, 2000.

[12] Jayaram K. Udupa, Punam K. Saha, "Fuzzy Connectedness and Image Segmentation", Proceedings of the IEEE, vol. 91, 2003.

[13] M Karnan, $\mathrm{R}$ Sivakumar, M Almelumangi, K Selvanayaki, T Logeswari, "Automatic Detection of the Suspicious Regions on Digital Brains Using Genetic Algorithm", Proceedings of National Conference and Workshop on Soft and Intelligent Computing, pp. 23-25, 2008.

[14] Y. Ge, Q. C. Meng, C. J. Yan, J. Xu, "A Hybrid Ant Colony Algorithm for Global Optimization of Continuous Multi-Extreme Functions", Proceedings of the Third International Conference on Machine Learning and Cybernetics, pp. 2427-2432, 2004.

[15] S Murugavallil, V Rajamani, "An Improved Implementation of Brain Tumor Detection Using Segmentation Based on Neuro Fuzzy Technique", Journal of Computer Science, vol. 3, no. 11, pp. 841846, 2007.

[16] P. Tamije;V. Palanisamy; T. Purusothaman: Performance Analysis of Clustering Algorithms in Brain Tumor Detectionof MR Images European Journal of Scientific Research, ISSN1450-216X Vol. 62 No. 3 2011, pp. 321 330.

[17] S. K. Bandyopadhyay and D. Saha, Brain region extraction volume calculation UNIASCIT, 1 , no. 1 , pp. 44-48, 2011.

[18] S. Datta; M. Chakraborty. Brain Tumor Detection from Pre-Processed MR Images using
SegmentationTechniques. Special Issue on 2nd National Conference-Computing, Communication and Sensor NetworkCCSN Published by Foundation of Computer Science, NewYork, USA. vol. 2, pp. 1-5, 2011.

[19] Kalaiselvi, T. and K. Somasundaram. Fuzzy c-means technique with histogram based centroid initialization forbrain tissue segmentation in MRI of head scans. IEEE International Symposium on Humanities, Science \&Engineering Research SHUSER, pp. 149-154, 2011.

[20] Janki Naik , Prof Sagar Patel , Tumor Detection and Classification using Decision Tree in Brain MRI, IJEDR, ISSN:2321-9939, 2013.

[21] K. Somasundaram, T. Kalaiselvi, Automatic brain extractionmethods for $\mathrm{T} 1$ magnetic resonance images using regionlabeling and morphological operations, ELSEVIER, Computersin Biology and Medicine 41 $2011716-725$

[22] E. A. El-Dihshan, T, Hosney, A B. M. Salem, Hybridinte lligence techniques for MRI Brain images classification,ELSEVIER, Digital Signal Processing, Volume 20, pp,433-441,2010

[23] Jin Liu, Min Li, Jianxin Wang, Fangxiang Wu, Tianming Liu, Yi Pan, A Survey of MR I-Based Brain Tumor Segmentation Methods Tsinghua Science and Technology, vol. 19, no. 6, December 2014.

[24] J. M. P. Gupta, M. M. Shringirishi, "Implementation of brain tumor segmentation in brain MR images using $\mathrm{k}$ means clustering and fuzzy c-means algorithm", International Journal of Computers \& Technology, vol. 5, no. 1, pp. 54-59, 2013.

[25] G.-C. Lin, W.-J. Wang, C.-C. Kang, C.-M. Wang, "Multispectral MR images segmentation based on fuzzy knowledge and modified seeded region growing", Magnetic Resonance Imaging, vol. 30, no. 2, pp. 230246, 2012.

[26] Prakash Mahindrakar, Dr. M. Hanumanthappa, "Data Mining in Healthcare: A Survey of Techniques and Algorithms with Its Limitations and Challenges", Int. Journal of Engineering Research and Applications, vol. 3, no. 6, pp. 937-941, Nov-Dec 2013, ISBN 2248-9622. 